\title{
Differences of Asphyxia in Infants of 35 and 36 Weeks Pregnant Women with or without Antenatal Corticosteroids
}

\author{
Udin Sabarudin*, Teuku Renardiansyah, Amillia Siddiq \\ Department of Obstetrics and Gynecology, Faculty of Medicine, University of Padjadjaran Dr. Hasan Sadikin \\ Hospital, Bandung, Indonesia \\ Email: *wg.repro.health@gmail.com
}

Received 17 April 2015; accepted 21 June 2015; published 24 June 2015

Copyright (C) 2015 by authors and Scientific Research Publishing Inc.

This work is licensed under the Creative Commons Attribution International License (CC BY). http://creativecommons.org/licenses/by/4.0/

cC) (i) Open Access

\begin{abstract}
Objective: To identify differences of Asphyxia in infants of 35 - 36 weeks with or without antenatal corticosteroid. Methods: Case control study was done on 35 and 36 weeks of pregnancy mother with threatened preterm labor who received and did not receive corticosteroids. Results: From 106 patients with threatened preterm labour between 35 - 36 gestational age, 53 patients received corticosteroid, and 53 did not receive corticosteroid. Incidence of asphyxia decreased significantly from patients received corticosteroid $(34 \%: 58.5 \%, P=0.011)$. Incidence of decreased in patients received corticosteroid compared with patients did not receive corticosteroid $(3.8 \%$ : 15.1\%), but the difference was not significant $(P=0.093)$. Based on the length of stay at the hospital, babies receiving corticosteroid before delivery have shorter duration of stay ( $<3$ days) with 94.3\%: 84.9\% ( $P=0.224)$. Conclusion: There is a significant correlation of newborn asphyxia from 35 - 36 gestational weeks with or without corticosteroid treatment $(58.5 \%: 34 \%, P=0.011)$.
\end{abstract}

\section{Keywords}

Corticosteroid, Premature, Asphyxia

\section{Introduction}

Prematurity is still a serious problem for all health workers around the world. Around 4 million neonates die because of prematurity [1]. Respiratory distress was associated with prematurity and the incidence was higher (29\%) than in term pregnancy (4\%). The major causes of respiratory distress are asphyxia, hyaline membrane disease

${ }^{*}$ Corresponding author.

How to cite this paper: Sabarudin, U., Renardiansyah, T. and Siddiq, A. (2015) Differences of Asphyxia in Infants of 35 and 36 Weeks Pregnant Women with or without Antenatal Corticosteroids. Open Journal of Obstetrics and Gynecology, 5, 360364. http://dx.doi.org/10.4236/ojog.2015.56052 
(HMD), transient tachypnea of the newborn, pneumonia, and pulmonary hypertension [2]. Respiratory distress syndrome is a serious complication on premature delivery that causes high neonatal mortality and morbidity rate. It is a disease of hypoventilation and a manifestation of pulmonary immaturity and surfactant deficiency. In respiratory distress syndrome, the lungs are poorly compliant with acinar atelectasis, and there is a gradual development of thickening of the interstitium and dilatation of the terminal airways. The incidence increased one fifth on low birth weight baby (BW $<2500 \mathrm{gr}$ ) and two third in very low birth weight baby $(<1500 \mathrm{gr})$ [3].

Respiratory failure in these infants was caused by insufficient surfactant production in lungs, immature lungs and other organs development [3]. Surfactant plays important roles in lungs development to prevent the alveoli to collapse during respiration [3]. Some studies said that antenatal corticosteroid administration could enhance fetal lung maturity [4].

Corticosteroid plays an important role in human organ development and differentiation at the third trimester. The use of corticosteroid was repeated if the delivery process did not proceed after the therapy was given. This statement was supported by another study which stated that the effect of corticosteroid would disappeared if the delivery process was longer than 7 days after corticosteroid therapy and the laboratory experiment confirmed that surfactant production could be induced more than once [4]. Corticosteroid administration could significantly decrease the risk of having intraventricular haemorrage from vasoconstrictor effect on fetal brain circulation. The recommended corticosteroid dosage was $12 \mathrm{mg}$ betamethasone every 24 hours for 2 doses or $6 \mathrm{mg}$ dexamethasone every 12 jam for 4 doses [5].

American Congress of Obstetricians and Gynaecologists (ACOG) published guidelines for corticosteroid administration is during 24 - 34 6/7 weeks with threatened premature delivery [5]. During January 2000 until December 2004, incidence of respiratory distress in premature neonates that receive corticosteroid compared with the premature nenonates that did not receive corticosteroid is $24 \%: 81.3 \%$ [6]. A study of 998 subjects stated that patients with corticosteroid administration more than 34 week pregnancy tended to have lower incidence of respiratory distress $(0.011 \%)$ than those who did not received the therapy $(0.002 \%)$ [7]. There is no study of corticosteroid effect during 34 weeks of gestational weeks. Based on these facts, researchers are interested to do the study about corticosteroid effect during 34 weeks of gestational age in Dr. Hasan Sadikin General Hospital, Bandung, West Java.

\section{Methods}

Case control study was done on pregnant mother of 35 - 36 weeks of gestational age with threatened premature delivery who received or not received corticosteroids. This study took place at Dr. Hasan Sadikin General Hospital Bandung, West Java, Indonesia during March 2014-May 2014.

Dr. Hasan Sadikin General Hospital Bandung is categorized as a level which means it is a province referral hospital. Total delivery rate during this study period is 3509 patients per year.

Sample taken from subjects qualified for the inclusion criteria. Sample size determined by using statistical calculation of $95 \%$ of confidence interval and $90 \%$ of power test then tested and analyzed using chi-square methods. By using the formula determining the sample size for analytical research categorical unpaired, then the sample size formula as follows:

$$
\begin{aligned}
& n_{1}=n_{2}=\left(\frac{Z_{\alpha} \sqrt{2 P Q}+Z_{\beta} \sqrt{P_{1} Q_{1}+P_{2} Q_{2}}}{P_{1}-P_{2}}\right)^{2} \\
& n=\frac{[1.96 \sqrt{2 \times 0.165 \times(1-0.165)}+1.64 \sqrt{0.29 \times(1-0.29)+0.04 \times(1-0.04)}]^{2}}{(0.29-0.04)^{2}}, n=52.17 \approx 52
\end{aligned}
$$

Minimum sample required for this study is 53 patients.

Samples are mother having 35 - 36 weeks of gestational age with threatened premature delivery during March 2014-Mei 2014 at Dr. Hasan Sadikin General Hospital Bandung, Indonesia. Patients were divided into two groups, group one given a full dose of corticosteroids (dexametasone $4 \times 6 \mathrm{mg}$ ) within two days, and group two didn’t receive any corticosteroid treatment prior to delivery. The outcome of the baby were examined by perinatologist for diagnosis of asphyxia. Diagnostic parameter defined by perinatology division. The research data then processed statistically. 
Inclusion criteria: Patients with 35 - 36 weeks of gestational age, singleton pregnancy with threatened premature delivery and planned for normal delivery, not getting antenatal corticosteroids before.

Exclusion criteria: Major congenital abnormalities in infants, incomplete medication of corticosteroids, premature rupture of membranes.

Variables which are used for this study are:

- Age: Maternal full year age during study period.

- Parity: The number of times a woman has given birth.

- APGAR score: Assessment method of the newborn to define whether a newborn is categorized as asphyxia or not asphyxia.

- Length of stay: Duration of a patient having treatment at the hospital.

Sample was taken during hospitalization, recorded in secondary data (medical record) and tertiary data (MCM, Maternal and Child Monitoring). The sample then analyzed using SPSS $23^{\text {rd }}$ edition.

\section{Results}

Table 1 shows that, based on age and parity, the two groups has similar general characteristics, which is indicated by value of $\mathrm{P}>0.05$. Thus the two groups were comparable.

Table 2 shows that babies did not received antenatal corticosteroid have higher incidence of having neonatal asphyxia (58.5\%:34\%, $\mathrm{P}=0.011)$. Based on $\mathrm{p}$ value, the result is statistically significant.

Table 3 shows that length of stay from the babies received antenatal corticosteroid is shorter than not received antenatal corticosteroid $(94.3 \%: 84.9 \%, \mathrm{P}=0.111)$. It is concluded that no significant correlation between antenatal corticosteroid with hospital length of stay.

Table 1. Characteristics of age and parity in maternal with 35 - 36 weeks of pregnancy, received and did not received corticosteroids.

\begin{tabular}{cccc}
\hline \multirow{2}{*}{ Characteristics } & Received corticosteroid & Not received corticosteorid & P value \\
\cline { 2 - 3 } & $\mathbf{N}=\mathbf{5 3}$ & $\mathbf{N}=\mathbf{5 3}$ & 0.403 \\
\hline Age & & & \\
$15-35$ years & $47(88.7 \%)$ & $44(83.0 \%)$ & 0.504 \\
$>35$ years & $6(11.3 \%)$ & $9(27.0 \%)$ & \\
Parity & $38(71.7 \%)$ & $41(77.4 \%)$ & $12(22.6 \%)$ \\
Nullipara & $15(28.3 \%)$ & & \\
Multipara & &
\end{tabular}

Table 2. Correlation of asphyxia to administration of antenatal corticosteroid in newborn babies.

\begin{tabular}{cccc}
\hline \multirow{2}{*}{ Variables } & Received corticosteroid & Not received corticosteroid & P value \\
\cline { 2 - 3 } & $\mathbf{m N}=\mathbf{5 3}$ & $\mathbf{N}=\mathbf{5 3}$ & $\mathbf{0 . 0 1 1}$ \\
\hline Incidence of asphyxia & & & \\
Asphyxia & $18(34.0 \%)$ & $31(58.5 \%)$ & $22(41.5 \%)$ \\
No asphyxia & $35(66.0 \%)$ & & \\
\hline
\end{tabular}

Table 3. Correlation length of stay to antenatal corticosteroid administration.

\begin{tabular}{ccc}
\hline Variables & Received corticosteroid & Not received corticosteroid \\
\cline { 2 - 3 } & $\mathbf{N}=\mathbf{5 3}$ & $\mathbf{N}=\mathbf{5 3}$ \\
\hline Length of stay & & $\mathbf{P}$ value \\
$<3$ days & $50(94.3 \%)$ & $45(84.9 \%)$ \\
$>3$ days & $3(5.7 \%)$ & $8(15.1 \%)$ \\
\hline
\end{tabular}




\section{Discussion}

Corticosteroid plays an important role in decreasing incidence of respiratory distress of the newborn [4]. In this study, evaluation was done on babies born from premature delivery that received antenatal corticosteroid during 35 - 36 gestational age. From 106 patients, there are 53 patients received antenatal corticosteroid and 53 did not received antenatal corticosteroid. Results showed that antenatal corticosteroid administration during 35 - 36 weeks of gestational age is significant to asphyxia incidence.

In 1972, a study using bethametasone was done and the result was it could decrease the incidence of respiratory distress as much as $25.8 \%$ in the control group compared to $9.0 \%$ in the group receiving therapy [8]. During 35 - 36 weeks, there are others factors related to respiratory distress etiology besides surfactant deficiency, corticosteroid administration was expected to form and exceeded the minimum threshold for the development of alveoli [9]. Newborns during this gestational age was unprepared physiological and metabolically. This will cause variety disorders in early stages of life [10]. Most common etiology for respiratory distress is Transient tachypnea of newborn as a result of lung fluid delayed absorption that cause the lung capacity to decrease [2]. Complications happens during this period will cause longer duration of treatment in the hospital, sometimes even intensive care unit is needed [10]. This is consistent with the recommendations issued by the ACOG that elective delivery therapy is not recommended in pregnancy under 39 weeks considering various morbidities may occur [11].

Other factors contributing to respiratory distress is the period of corticosteroid administration. Patients having delivery in 24 - 72 hours during complete dose of antenatal corticosteroid had better outcome than patients having deliveries more than 72 hours after corticosteroid administration [4]. The administration of corticosteroids besides accelerating the growth of the lung, have other effects that would arise at the age of 2 years, it may resulting a neurological disorders or insulin resistance. A research followed the development of children who received corticosteroids mentioned that incidence of cerebral palsy, blindness, hearing loss and cognition was increased at 2 years old [12]. In addition, other study that followed up until the early days of elementary school mentioned there is attention disorders in children received antenatal corticosteroid [13]. Limitations of this study was not able to follow the late side effects that may arise in the administration of corticosteroids at 36 - 36 weeks gestation because of time limitation. Currently administration of corticosteroids protocol in Dr. Hasan Sadikin General Hospital refers to the protocol issued by ACOG, it is given at the age of 24 pregnancies and 33 6/7 weeks. With this study it can be concluded that the protocol can be adjusted for corticosteroids in Dr Hasan Sadikin General Hospital because there are significant advantages in administration of corticosteroids in pregnancy over 34 weeks.

Study limitation during this study is lack of time to do the follow up to the babies with asphyxia. Loss of follow up to the neonates also caused by lack of discipline from the mother to visit the clinics for follow up after hospital discharge.

\section{Conclusion}

There is a significant correlation between antenatal corticosteroid administration during 35 - 36 weeks of gestational age and the incidence of asphyxia of the newborn.

\section{References}

[1] Kambafwile, J.M., Cousens, S., Hansen, T. and Lawn, J.E. (2010) Antenatal Steroids in Preterm Labour for the Prevention of Neonatal Deaths Due to Complications of Preterm Birth. International Journal of Epidemiology, 39, 11221133.

[2] Porto, A.M., Coutinho, I.C., Correia, J.B. and Amorim, M.M. (2011) Effectiveness of Antenatal Corticosteroids in Reducing Respiratory Disorders in Late Preterm Infants: Randomised Clinical Trial. BMJ, 342, d1696. http://dx.doi.org/10.1136/bmj.d1696

[3] Dalziel, R.D. (2013) Antenatal Cortocosteroids for Accelerating Fetal Lung Maturation for Women at Risk of Preterm Birth (Review). Cochrane Collaboration, 3.

[4] McKinlay, C.J., Crowther, C.A., Middleton, P. and Harding, J.E. (2012) Repeat Antenatal Glucocorticoids for Women at Risk of Preterm Birth: A Cochrane Systematic Review. American Journal of Obstetrics Gynecology, 206, 187-194. http://dx.doi.org/10.1016/j.ajog.2011.07.042

[5] Bastek, J.A., Langmuir, H., Kondapalli, L.A., Pare, E., Adamczak, J.E. and Srinivas, S.K. (2012) Antenatal Cortico- 
steroids for Late-Preterm Infants: A Decision-Analytic and Economic Analysis. ISRN Obstetrics and Gynecology, 2012, Article ID: 491595. http://dx.doi.org/10.5402/2012/491595

[6] Ventolini, G., Neiger, R., Mathews, L., Adragna, N. and Belcastro, M. (2008) Incidence of Respiratory Disorders in Neonates Born between 34 and 36 Weeks of Gestation Following Exposure to Antenatal Corticosteroids between 24 and 34 Weeks of Gestation. American Journal of Perinatology, 2, 79-83. http://dx.doi.org/10.1055/s-2007-1022470

[7] Stutchfield, P., Whitaker, R. and Russell, I. (2005) Antenatal Betamethasone and Incidence of Neonatal Respiratory Distress after Elective Caesarean Section: Pragmatic Randomised Trial. BMJ, 331, 662. http://dx.doi.org/10.1136/bmj.38547.416493.06

[8] Liggins, G.C. and Howie, R.N. (1972) A Controlled Trial of Antepartum Glucocorticoid Treatment for Prevention of the Respiratory Distress Syndrome in Premature Infants. Pediatrics, 50, 515-525.

[9] Wapner, R. and Jobe, A.H. (2011) Art and Science, Clinics in Perinatology. Clinics in Perinatology, 38, 529-545.

[10] Mumdzhiev, H. (2012) The Late Preterm Infants—Time to Put Our Mind. Akush Ginekol (Sofiia), 51, 38-45.

[11] Ashton, D.M. (2010) Elective Delivery at Less than 39 Weeks. Current Opinion in Obstetrics and Gynecology, 22, 506-510. http://dx.doi.org/10.1097/GCO.0b013e3283404eb4

[12] Asztalos, E. (2012) Antenatal Corticosteroids: A Risk Factor for the Development of Chronic Disease. Journal of Nutrition and Metabolism, 2012, Article ID: 930591. http://dx.doi.org/10.1155/2012/930591

[13] Talge, N.M., Holzman, C., Van Egeren, L.A., Symonds, L.L., Scheid, J.M., Senagore, P.K., et al. (2012) Late-Preterm Birth by Delivery Circumstance and Its Association with Parent-Reported Attention Problems in Childhood. Journal of Developmental Behavioral Pediatrics, 33, 405-415. http://dx.doi.org/10.1097/DBP.0b013e3182564704 